\title{
The nature of dwarf nova outbursts
}

\author{
V. Buat-Ménard ${ }^{1}$, J.-M. Hameury ${ }^{1}$, and J.-P. Lasota² \\ 1 Observatoire de Strasbourg, UMR 7550 du CNRS, 11 rue de l'Université, 67000 Strasbourg, France \\ e-mail: hameury@astro.u-strasbg.fr \\ 2 Institut d'Astrophysique de Paris, 98bis Boulevard Arago, 75014 Paris, France \\ e-mail: lasota@iap.fr
}

Received 11 October 2000 / Accepted 14 November 2000

\begin{abstract}
We show that if the dwarf-nova disc instability model includes the effects of heating by stream impact and tidal torque dissipation in the outer disc, the calculated properties of dwarf-nova outbursts change considerably, and several notorious deficiencies of this model are repaired. In particular: (1) outside-in outbursts occur for mass transfer rates lower than in the standard model as required by observations; (2) the presence of long (wide) and short (narrow) outbursts with similar peak luminosities is a natural property of the model. Mass-transfer fluctuations by factors $\sim 2$ can explain the occurrence of both long and short outbursts above the cataclysmic variable period gap, whereas below $2 \mathrm{hr}$ only short normal outbursts are expected (in addition to superoutbursts which are not dealt with in this article). With additional heating by the stream and tidal torques, such fluctuations can also explain the occurrence of both outside-in and inside-out outbursts in SS Cyg and similar systems. The occurrence of outside-in outbursts in short orbital-period, low mass-transfer-rate systems requires the disc to be much smaller than the tidal-truncation radius. In this case the recurrence time of both inside-out and outside-in outbursts have a similar dependence on the mass-transfer rate $\dot{M}_{2}$.
\end{abstract}

Key words. accretion, accretion discs - instabilities - (stars:) novae, cataclysmic variables - (stars:) binaries: close

\section{Introduction}

Dwarf novae (DN) are erupting cataclysmic variable stars. The 4-6 mag outbursts last from few days to more than a month and the recurrence times can be as short as a few days and as long as 30 years (see e.g. Warner 1995). In cataclysmic variables, a Roche-lobe filling low-mass secondary star is losing mass which is accreted by a white dwarf primary. In DNs the matter transfered from the secondary forms an accretion disc around the white dwarf; it is this disc which is the site of the outbursts. It is believed that an accretion disc instability due to partial hydrogen ionization is triggering the outbursts. The disc instability model (DIM; see Cannizzo 1993; Lasota 2000 for reviews) is supposed to describe the whole DN outburst cycle. This model assumes the $\alpha$-prescription (Shakura \& Sunyaev 1973) for the viscous heating and the angular momentum transport in the disc. In such a framework, the outbursts are due to propagating heating and cooling fronts, while during quiescence the disc is refilling the mass lost during the outburst. In the standard version of the model, the mass transfer rate is assumed to be constant during the whole outburst cycle. In order to reproduce outburst amplitudes and durations, the

Send offprint requests to: V. Buat-Ménard, e-mail: buat@astro.u-strasbg.fr $\alpha$-parameter has to be larger in outburst than in quiescence (Smak 1984).

The DIM is widely considered to be the model of DN outbursts because it identifies the physical mechanism giving rise to outbursts, and with the above mentioned assumptions it produces outburst cycles which roughly look like the real thing. A closer look, however, shows that the model fails to reproduce some of the fundamental properties of dwarf-nova outbursts. The main deficiencies of the DIM were recently summarized by Smak (2000). First, models predict a significant increase of the disc luminosity during quiescence (see e.g. Fig. 3), an effect which is not observed. Second, in many systems the distribution of burst widths is bimodal (van Paradijs 1983). The DIM can reproduce the width bimodality but, contrary to observations, its narrow outbursts have lower amplitudes than the wide ones. Third, observations clearly show that during outbursts the secondary is irradiated and the mass transfer rate increases. The standard DIM does not take this into account. To these problems pointed out by Smak one can add several other deficiencies. The model predicts outbursts beginning in the outer disc regions ("outside-in") only for high mass transfer while observations show the presence of such outbursts at apparently low mass transfer rates. In most cases the model predicts quiescent accretion rate which are at least two orders of magnitude lower than the rates deduced from quiescent luminosities. Finally, it 
is clear that superoutbursts observed in SU UMa stars and other systems (Smak 2000) cannot be explained by the standard DIM.

All this leads to the obvious conclusion that the standard version of the DIM has to be enriched by inclusion of physical processes which might be important but which, for various reasons, were not taken into account in the original model. Osaki (1989) proposed that superoutbursts are due to a tidal-thermal instability. Smak (2000) suggested that problems of this model could be solved by a hybrid which would combine it with the radiation-induced enhanced mass transfer model (Osaki 1985; Hameury et al. 1997). He included mass transfer enhancements during outburst into the standard model and suggested that this could explain properties of the narrow and wide outbursts which alternate in many systems (Smak 1999a). Hameury et al. (2000), extending previous work by Hameury et al. (1997) and Hameury et al. (1999), showed that inclusion of effects such as mass transfer variations, formation of holes in the inner disc and irradiation of both the disc and the secondary allows to reproduce many properties of the observed outbursts.

Some questions, however, were left without satisfactory answers. First, the problem of "outside-in" outbursts occurring for too high mass-transfer rates was still unsolved. Second, Smak (1999a) obtained narrow and wide outbursts by including irradiation induced mass transfer fluctuations but wide outbursts in his model result from fluctuations whose amplitude bring the disc to a stable state. In such a case the width of wide outbursts would not be determined by the disc structure alone. There is nothing fundamentally wrong with such a model and long standstills of Z Cam systems are most probably due to such fluctuations, as proposed by Meyer \& Meyer-Hofmeister (1983) and shown by Buat-Ménard et al. (2000). However, one should check that the model is complete, that no other mechanism can explain outburst width distribution.

In the present paper we study two mechanisms which dissipate energy in the outer parts of an accretion disc. They are: (1) the impact on the outer boundary of the accretion disc of the gas stream which leaves the secondary through the $L_{1}$ critical point of the Roche lobe; this impact zone is observed in the light-curves as a bright ("hot") spot; (2) the viscous dissipation induced by the tidal torques which are responsible for the disc outer truncation. These mechanisms which may heat up the disc's outer regions have been known for a long time (Paczyński 1977; Papaloizou \& Pringle 1977; Meyer \& Meyer-Hofmeister 1983), but have been neglected in most of DIM calculations. The exception is Ichikawa \& Osaki (1992) who included both effects in their model. They used, however, a viscosity prescription specially designed to suppress inside-out outbursts and did not perform a systematic study of the effects as a function of the mass-transfer rate, size of the disc etc.

We add stream-impact and tidal torque heating effects to the Hameury et al. (1998) version of the DIM, presented in Sect. 2. We use a 1D approximation because the dynamical time-scale is shorter than the thermal time-scale. We study the effect of the stream impact (Sect. 3) and the tidal torques (Sect. 4) on outburst properties in short and long period DN systems. Both effects change the energy equation of the standard DIM. In both cases we find similar results: when compared to the standard case, "outsidein" outbursts occur for lower mass transfer rate. The recurrence time is decreased by both the stream impact and dissipation of the tidal torques. We conclude, therefore, that heating of the outer disc by the stream impact and the rate of work by tidal torques provides a satisfactory explanation of the presence of "outside-in" outbursts at realistic mass-transfer rates.

The most important result of our investigation is that when these effects are included one can obtain two types of outbursts: short ("narrow") and long ("wide") (Sect. 5). We find a critical mass transfer rate for which these two type of outburst "alternate". This mass transfer rate is very close to that deduced from evolutionary calculations for systems with orbital periods longer than 3 hours ("above the period gap"). For lower mass-transfer rates only narrow outbursts are present; for higher ones, outbursts are only of the wide type. Fluctuations of the masstransfer rate allow to expand the range of orbital periods for which the bimodal outburst width distribution is occurring. We conclude that the observed bimodal outburst width distribution is caused by the outer-disc heating.

In Sect. 6 we discuss conditions for which outside-in outbursts can occur. We show that one can naturally account for the occurrence of both types of outbursts in SS Cyg if the mass transfer rate fluctuates by factors $\lesssim 2$ around a mean value which is close to what is generally assumed as the average mass transfer rate in this system. Outside-in outbursts in systems below the period gap, which have low mass transfer rates, can be explained only if the disc is significantly smaller than the tidal truncation radius. We show that this is in agreement with observations and consistent with expectations of the tidal-thermal instability model, at least during the first half of the supercycle of SU UMa systems. We also point out the ambiguity in observational determination of outburst "outside-in" or "inside-out" type, also respectively called A and B types. These types are generally assigned to outbursts according to their shape and the UV delay. We question the general validity of such criteria and argue that the eclipse profile observations during outbursts is the only reliable diagnostics of the outburst type.

\section{The model}

We use the recent version of the disc instability model described in Hameury et al. (1998). We assume here that $\alpha=$ $\alpha_{\text {cold }}=0.04$ in quiescence and $\alpha=\alpha_{\text {hot }}=0.2$ in outburst (Smak 1999b). Difficulties in reproducing the observed UV delays (see Sect. 6 for more details) and accounting for observed quiescent X-ray fluxes well above the values predicted by the DIM, led to the suggestion that "holes" are 
present in the center of quiescent accretion discs (Meyer \& Meyer-Hofmeister 1994; Lasota et al. 1995). Smak (1998) pointed out, however, that the failure to reproduce observed UV delays by numerical codes was largely due to the assumption of a fixed outer disc radius, which prevented the occurrence of outside-in outbursts. In addition, the discs used in such calculations were too small to give the required time-scales. When one uses reasonable outer boundary conditions, long UV delays can be observed. However, if one wishes to reproduce both the delays and the light-curve shapes, a truncated disc might be still a necessary ingredient of the model (Hameury et al. 1999). Such a hole could be due to magnetic disruption in the case of a magnetic white dwarf or to evaporation in the general case. Because we are not interested here in the exact values of the rise time and the UV delay, we use a fixed size hole only for numerical convenience. The inner disc radius is fixed at $r_{\text {in }}=10^{9} \mathrm{~cm}$, while $510^{8} \mathrm{~cm}$ is approximately the radius of a $1.2 M_{\odot}$ white dwarf and $6.910^{8} \mathrm{~cm}$ correspond to the other mass we use: $0.8 M_{\odot}$. The outer boundary condition depends on the circularization radius $r_{\text {circ }}$ at which a particle that leaves the Lagrangian point $L_{1}$ with angular momentum $j=\Omega_{\text {orb }} b^{2}$ (where $\Omega_{\text {orb }}$ is the orbital angular velocity and $b$ the distance between the primary and the $L_{1}$ point), would stay in circular orbit if there were no accretion disc. From $j=\Omega_{\mathrm{K}} r_{\text {circ }}^{2}$, where $\Omega_{\mathrm{K}}$ is the Keplerian angular frequency, one gets: $r_{\text {circ }} \simeq \Omega_{\text {orb }}^{2} b^{4} / G M$. When one takes the gravitational potential of the secondary into account, these relations are slightly modified, and we linearly interpolate the table from Lubow \& Shu (1975) to calculate $r_{\text {circ }}$. The mean outer radius $\left\langle r_{\text {out }}\right\rangle$ is taken to be the average of the three radii $r_{1}, r_{2}$ and $r_{\max }$ calculated in Table 1 of Paczyński (1977).

We consider in this paper the effects of the impact of the gas stream coming from the secondary (Sect. 3) and of the dissipation induced by tidal torques (Sect. 4) in both short and long period dwarf novae (thus for both small and large accretion discs) using two characteristic sets of parameters taken from Ritter \& Kolb (1998): those of SU UMa and SS Cyg (Table 1). For the short period systems, although we take the parameters of SU UMa, we include neither the tidal instabilities (suggested by Osaki (1989) to be responsible for superoutbursts in SU UMa), nor the combination of irradiation, mass transfer variation and evaporation used by Hameury et al. (2000) to obtain long lasting outbursts. Therefore, we do not expect to reproduce superoutbursts. We also neglect irradiation of secondary star which in principle may have significant impact on some of our results. We will consider this effect in a future work.

\section{Stream impact}

\subsection{Description}

The gas stream from the secondary hits the accretion disc creating the observed bright spot. As the dynamical time
Table 1. Parameters adopted for long and short period systems

\begin{tabular}{lcc}
\hline & Long period & Short Period \\
\hline$M_{1} / M_{\odot}$ & 1.2 & 0.8 \\
$M_{2} / M_{\odot}$ & 0.7 & 0.15 \\
$P_{\text {orb }}(\mathrm{hr})$ & 6.6 & 1.83 \\
$r_{\text {circ }}\left(10^{10} \mathrm{~cm}\right)$ & 1.14 & 0.91 \\
$<r_{\text {out }}>\left(10^{10} \mathrm{~cm}\right)$ & 5.4 & 2.3 \\
\hline
\end{tabular}

is smaller than the thermal time $\left(t_{\mathrm{dyn}} / t_{\mathrm{th}}=\alpha<1\right)$, the temperature is roughly constant around each annulus of the accretion disc. Thus, the effect of stream impact could be approximatively axisymmetric and we can use a 1D approximation. This assumption is obviously very rough. Spruit \& Rutten (1998) have shown that the stream impact region in WZ Sge is not at all axisymmetric (see also Marsh \& Horne 1990 for IP Peg). Moreover, if we refer to the generally assumed values of $\alpha, t_{\mathrm{dyn}} / t_{\mathrm{th}}$ is not that small in outburst; in addition, as the hot spot temperature $T_{\mathrm{hs}} \gg T_{\text {disc }}$ in the outer layers, the actual thermal time is much smaller than the unperturbed equilibrium value. Finally, we do not know exactly how the stream impact interacts with the disc and where precisely its energy is deposited in the disc; e.g. in the simulations of Armitage \& Livio $(1996,1998)$ a significant amount of the stream material can ricochet off the disc edge and overflow toward smaller radii.

It is therefore difficult to model properly the hot-spot contribution to the energy balance of the outer layers of an accretion disc. Here we consider that the stream impact heats an annulus $\Delta r_{\mathrm{hs}}$ of the disc with an "efficiency" $\eta_{\mathrm{i}}$. The coefficient $\eta_{\mathrm{i}}$ is supposed to represent the rather complex way the outer disc is heated by the stream. The energy per unit mass released in the shock between the stream and the disc is $\left(\boldsymbol{V}_{2}-\boldsymbol{V}_{1}\right)^{2}$, where $\boldsymbol{V}_{2}$ is the Keplerian velocity at the outer disc edge, $\left|\boldsymbol{V}_{2}\right|=$ $G M_{1} /\left(2 r_{\text {out }}\right)$, and $\boldsymbol{V}_{1}$ is the stream velocity at the position of impact. As the angle between $\boldsymbol{V}_{1}$ and $\boldsymbol{V}_{2}$ is large, and $\left|\boldsymbol{V}_{1}\right|$ is comparable to $\left|\boldsymbol{V}_{2}\right|,\left|\boldsymbol{V}_{2}-\boldsymbol{V}_{1}\right|$ is of order of $G M_{1} /\left(2 r_{\text {out }}\right)$.

The heating rate $Q_{\mathrm{i}}$ is therefore taken as:

$Q_{\mathrm{i}}(r)=\eta_{\mathrm{i}} \frac{G M_{1} \dot{M}_{2}}{2 r_{\mathrm{out}}} \frac{1}{2 \pi r_{\mathrm{out}} \Delta r_{\mathrm{hs}}} \exp \left(-\frac{r_{\mathrm{out}}-r}{\Delta r_{\mathrm{hs}}}\right)$

where $M_{1}$ is the primary's mass, $\dot{M}_{2}$ the mass transfer rate from the secondary and $r_{\text {out }}$ the accretion disc outer radius. This assumes that the difference between the stream and the Keplerian kinetic energy is released in a layer of width $\Delta r_{\text {hs }}$ with an exponential attenuation. Note that $\eta_{\mathrm{i}}$ can be larger than unity, as $\left|\boldsymbol{V}_{2}-\boldsymbol{V}_{1}\right|$ can be larger than the Keplerian velocity. In the following, we take $\eta_{\mathrm{i}}=1$.

The energy equation then becomes:

$$
\begin{aligned}
\frac{\partial T_{\mathrm{c}}}{\partial t}= & \frac{2\left(Q^{+}+1 / 2 Q_{\mathrm{i}}-Q^{-}+J\right)}{C_{\mathrm{P}} \Sigma}-\frac{\mathcal{R} T_{\mathrm{c}}}{\mu C_{\mathrm{P}}} \frac{1}{r} \frac{\partial r v_{r}}{\partial r} \\
& -v_{r} \frac{\partial T_{\mathrm{c}}}{\partial r} .
\end{aligned}
$$


Here, $T_{\mathrm{c}}$ is the central temperature, $Q^{+}=(9 / 8) \nu \Sigma \Omega_{\mathrm{K}}^{2}$ and $Q^{-}=\sigma T_{\text {eff }}^{4}$ the heating and cooling rate respectively, $\Sigma$ the surface density, $\nu$ the kinematic viscosity coefficient, $J$ is a term that accounts for the radial energy flux carried either by viscous processes or by radiation (see Hameury et al. 1998), and $v_{r}$ is the radial velocity. $C_{\mathrm{P}}$ is the specific heat at constant pressure, $\mu$ the mean molecular weight, and $\mathcal{R}$ the perfect gas constant.

The cooling rate $Q^{-}\left(\Sigma, T_{\mathrm{c}}, r\right)$ is obtained from interpolation in a grid obtained by solving the vertical structure of the disc (Hameury et al. 1998). These structures are calculated assuming a steady state, the departure from thermal equilibrium being accounted for by an effective viscosity parameter $\alpha_{\text {eff }}$ different from the actual viscosity parameter $\alpha$. We assume here that the effect of the stream impact on the vertical structure can also be accounted for in this way. This would be perfectly correct if the local heating term were proportional to pressure, as is viscosity; if on the other hand it were localized at the disc surface, the problem would resemble that of disc illumination, and also in such a case, the use of an effective $\alpha_{\text {eff }}$ leads to qualitatively correct results: Stehle \& King (1999), who used this approximation, and Dubus et al. (1999), who calculated the exact vertical structure of irradiated discs, obtained similar modifications of the $\mathrm{S}$ curves by irradiation of the disc.

\subsection{Results}

Figure 1 displays the $\Sigma-T_{\text {eff }}$ curves, calculated at two different radii with and without the heating by the stream impact. These are the well known "S-curves" in which three branches can be distinguished: the stable, low- and high-state branches where $T_{\text {eff }}$ increases with $\Sigma$, and the unstable middle branch where $T_{\text {eff }}$ decreases with $\Sigma$ increasing. The latter branch is delimited by two critical points $\left(\Sigma_{\min }, T_{\min }\right)$ and $\left(\Sigma_{\max }, T_{\max }\right)$. To compute these S-curves, a steady state was assumed so $T_{\text {eff }}$ is proportional to $\dot{M}^{1 / 4}$. As mentioned above, we take $\eta_{\mathrm{i}}=1$.

When stream impact is taken into account one observes at large radii significant deviations from the standard case. It must, however, be stressed that $Q_{\mathrm{i}}$ is a nonlocal quantity proportional to $\dot{M}_{2}$ and thus depending on the outer boundary condition, whereas the usual S-curves are locally defined. Hence, the interpretations of these curves is not straightforward. For a fixed $\Sigma$, there is a factor $\Delta \log T_{\text {eff }} \sim 0.5$ between curves 2 and $2^{\prime}$. For a fixed $T_{\text {eff }}, \Sigma$ is reduced in the outer disc region when heating by the stream impact is included. It should therefore be easier, for a fixed mass transfer rate, to trigger outbursts starting at the outer edge of the disc when heating by the stream impact is included. One also expects the disc to be less massive. Moreover, the $\Sigma_{\min }-\Sigma_{\max }$ interval decreases (see Fig. 2) so that the recurrence time should decrease too.

Figure 3 shows light curves calculated without and with heating for a long period system ("SS Cyg"). One can

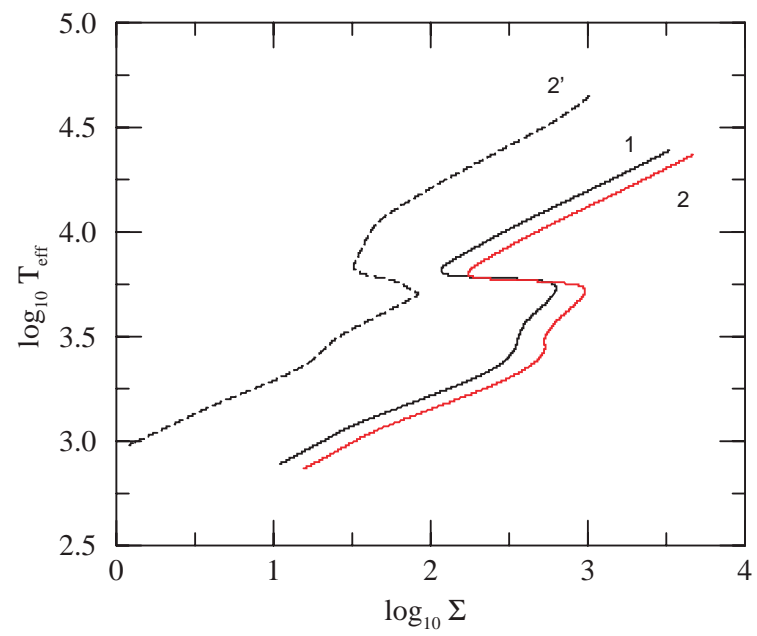

Fig. 1. $\Sigma-T_{\text {eff }}$ curves for the parameters of SS Cyg. Curve 1 is calculated at a radius $3.7510^{10} \mathrm{~cm}$ that is out of the stream impact region. Curves $2^{\prime}$ and 2 are calculated at a radius $5.210^{10} \mathrm{~cm}$ with and without the stream impact included in the model. For the hot branch $\alpha_{\text {hot }}=0.2$, for the cold one $\alpha_{\text {cold }}=0.04$. The S-curve is obtained using Eq. (40) in Hameury et al. (1998)

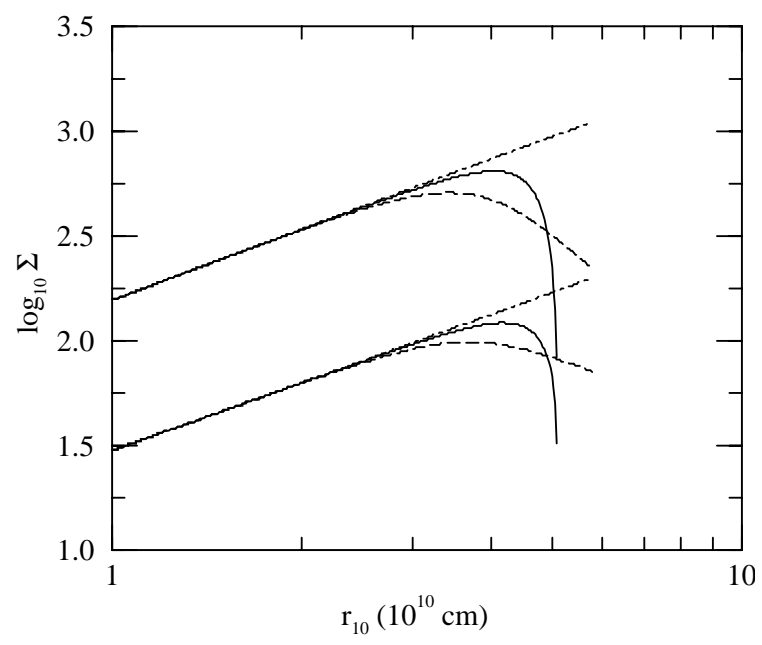

Fig. 2. $\Sigma_{\min }$ (lower curves) and $\Sigma_{\max }$ (upper curves) for SS Cyg parameters. The dot-dashed lines show the results of the standard DIM, the solid lines have been obtained when the stream impact is taken into account, and the dashed lines when tidal effects are included

see that the recurrence time is reduced in the model with the stream impact and that the quiescent luminosity is increased, as expected, since the stream impact heats up the outer parts of the disc which dominate the quiescent luminosity. During quiescence, however, the luminosity still increases in contrary to observations. Figures 4 and 5 show how the outburst type and the recurrence times depend on the mass-transfer rate. Including the additional heating by the stream impact implies that outside-in outbursts are triggered for lower mass transfer rates than in the standard DIM. Also the upper critical rate $\dot{M}_{\mathrm{c}}$, above which the disc is in a steady hot state, is lower as predicted by Meyer \& Meyer-Hofmeister (1983). 


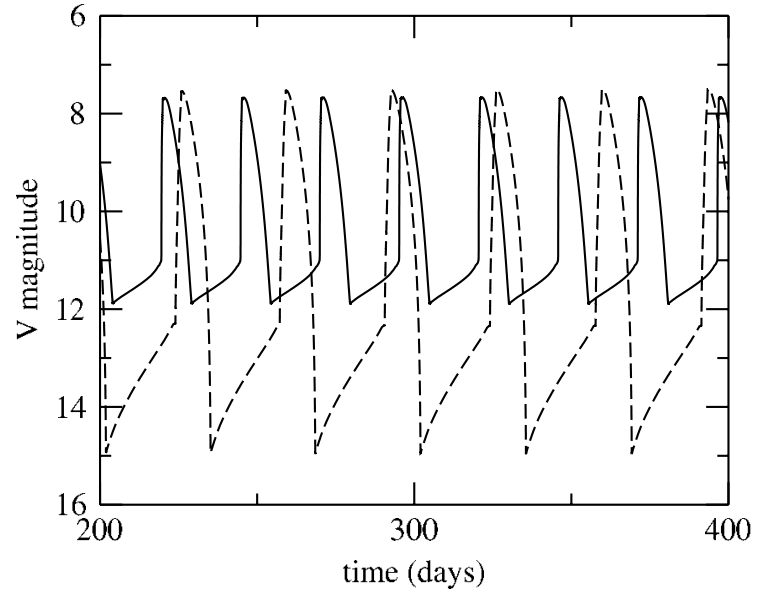

Fig. 3. Comparison of light curves with (solid line) and without the stream impact effect (dashed line). The parameters we use are taken from Table 1 and $\dot{M}_{2}=210^{17} \mathrm{~g} \mathrm{~s}^{-1}, \eta_{\mathrm{i}}=1$ and $\Delta r_{\mathrm{hs}}=r_{\mathrm{out}} / 10$

For larger $\Delta r_{\mathrm{hs}}$, the quiescent luminosity is smaller, because the additional amount of energy per unit surface decreases in the outer parts of the disc (note also that the integral of $Q_{\mathrm{i}}$ over the disc surface depends weakly on $\Delta r_{\mathrm{hs}}$ because of the simplified form of Eq. (1) where terms of order of $\Delta r_{\mathrm{hs}} / r_{\text {out }}$ have been neglected). For smaller $\eta_{\mathrm{i}}$, the recurrence time and the quiescent magnitude are higher. As short period systems have small discs, one might have expected that the stream impact would have stronger effects in these systems, but the results are really similar as can be seen in Figs. 4 and 5. However, in these calculations we assumed that the average disc outer radius is equal to the tidal radius. This is an overestimate of the disc's size, especially for the SU UMa stars. Realistically small discs will be discussed in Sect. 6 .

The main effects of the stream-impact heating seen on these figures are: $(1)$ the critical accretion rate $\dot{M}_{\mathrm{c}}\left(\Sigma_{\text {min }}\right)$ above which the disc is stable, is decreased by a factor of more than 3; (2) the transition between inside-out and outside-in outburst ("A-B transition") occurs for a transfer rate $\dot{M}_{\mathrm{A}-\mathrm{B}}$ that is decreased by a factor $\leq 2 ;(3)$ the recurrence time is almost unchanged. As expected (Osaki 1996), the quiescence time is approximately constant when the outbursts are of the inside-out type, and decreases (very roughly as $\dot{M}_{2}^{-2}$ ) for outside-in outbursts, although for the smaller disc some deviations from this relation can be seen (see Sect. 6). (Note that we plot here the time in quiescence and not the recurrence time.)

For systems with parameters close to that of SS Cyg, the inclusion of the stream impact heating lowers the critical rate for outside-in outburst from $\dot{M}_{\mathrm{A}-\mathrm{B}} \simeq$ $2.510^{17} \mathrm{~g} \mathrm{~s}^{-1}$ to $\dot{M}_{\mathrm{A}-\mathrm{B}} \simeq 1.710^{17} \mathrm{~g} \mathrm{~s}^{-1}$. The quiescence time is also shorter but only by a few days as long as $\dot{M}_{2}$ is not too close to $\dot{M}_{\text {c }}$ (see Fig. 4).

For short period systems, the critical mass transfer is $\dot{M}_{\mathrm{c}} \simeq 510^{16} \mathrm{~g} \mathrm{~s}^{-1}$, however, the transition between insideout and outside-in outbursts still occurs for mass-transfer rates $\left(\dot{M}_{\mathrm{A}-\mathrm{B}} \simeq 210^{16} \mathrm{~g} \mathrm{~s}^{-1}\right)$ much higher that what is

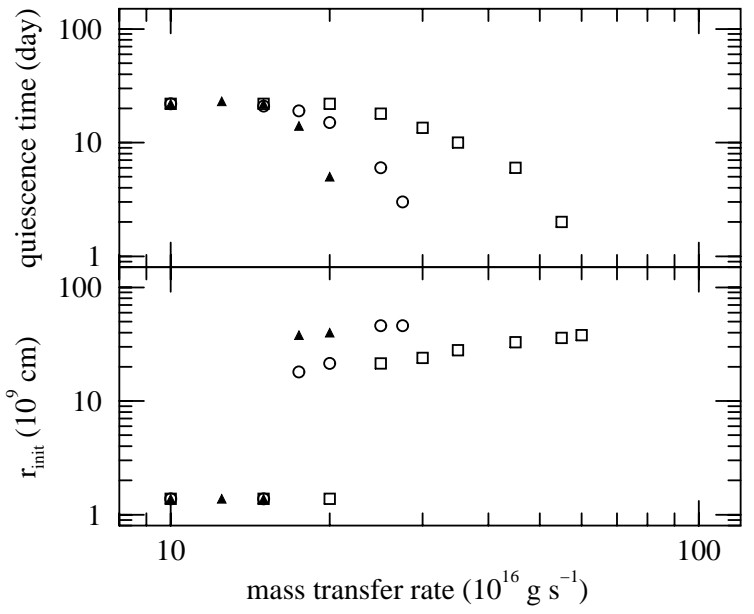

Fig. 4. Radius $r_{\text {init }}$ at which the instability is triggered and quiescence time as a function of the mass transfer rate from the secondary. The parameters are those of SS Cyg. Symbols correspond to the following: squares for the standard DIM; circles when the stream impact is included; triangles when the stream impact and the tidal dissipation are both included. The high mass-transfer end of each sequence of symbols corresponds approximately to the stability limit

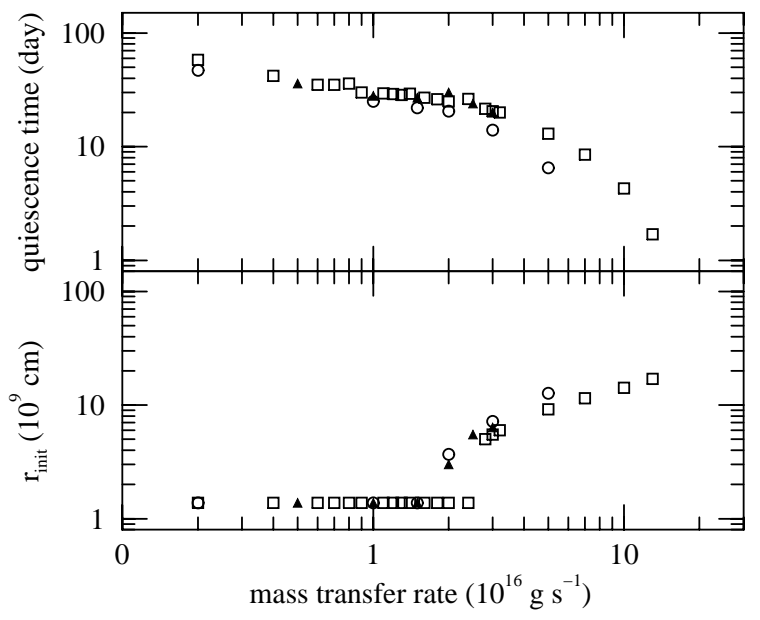

Fig. 5. Same as Fig. 4 for short period systems

estimated for these systems (less than a few $\times 10^{15} \mathrm{~g} \mathrm{~s}^{-1}$; see e.g. Warner 1995). We will discuss this problem in Sect. 6

\section{Tidal dissipation}

\subsection{Description}

Accretion discs in close binary systems are truncated by the tidal effect of the secondary star at a radius close to the Roche radius, the so-called tidal truncation radius: angular momentum transported outwards by viscous torques is returned to the binary orbital motion by the tidal torques acting on the disc's outer edge. In the standard DIM model, tidal torques can be included in the 
angular momentum equation in the following way (Smak 1984; Hameury et al. 1998):

$\frac{\partial \Sigma}{\partial t}=-\frac{1}{r} \frac{\partial}{\partial r}\left(-\frac{3}{2} r^{2} \Sigma \nu \Omega_{\mathrm{K}}\right)-\frac{1}{2 \pi r} T_{\mathrm{tid}}$

where the tidal torque per unit of area can be written as

$T_{\mathrm{tid}}=\operatorname{cr} \nu \Sigma \Omega_{\mathrm{orb}}\left(\frac{r}{a}\right)^{n}$

where $a$ is the orbital separation of the binary and $c$ a numerical constant that allows to adjust the tidal truncation radius $\left.<r_{\text {out }}\right\rangle$ that the disc would have had if it were steady (this gives an average value of the outer radius). The $r^{n}$ term used in this formula determines the disc fraction that is significantly affected by the tidal-torque dissipation (it corresponds to radii where the $\Sigma_{\min }$ and $\Sigma_{\max }$ curves are decreasing with radius (Fig. 2)). We use $n=5$ which is quite an uncertain value, and we discuss in Sect. 6 the effects of changing it.

As pointed out by Smak (1984) and discussed in detail by Ichikawa \& Osaki (1994), tidal torques (as viscous torques) induce a viscous dissipation (Papaloizou \& Pringle 1977):

$Q_{\mathrm{tid}}=\left(\Omega_{\mathrm{K}}-\Omega_{\mathrm{orb}}\right) T_{\mathrm{tid}}$

This tidal dissipation is usually neglected as it is small compared to $Q^{+}$except near the outer radius. During outbursts, the disc radius varies significantly, so does the tidal-torque dissipation which can be large enough to modify the outburst behaviour. We therefore include this term in the energy equation:

$$
\begin{aligned}
\frac{\partial T_{\mathrm{c}}}{\partial t}= & \frac{2\left(Q^{+}+1 / 2 Q_{\mathrm{tid}}+1 / 2 Q_{\mathrm{i}}-Q^{-}+J\right)}{C_{\mathrm{P}} \Sigma} \\
& -\frac{\mathcal{R} T_{\mathrm{c}}}{\mu C_{\mathrm{P}}} \frac{1}{r} \frac{\partial r v_{\mathrm{r}}}{\partial r}-v_{\mathrm{r}} \frac{\partial T_{\mathrm{c}}}{\partial r} .
\end{aligned}
$$

\subsection{Standard DIM plus tidal dissipation}

We first discuss the tidal effect alone, without including the heating by the stream impact. We find that type A outbursts are obtained for lower mass transfer rates than in the standard case; $\dot{M}_{\mathrm{A}-\mathrm{B}}$ is only slightly larger than in the case where the stream impact is included. For long period systems, we found $\dot{M}_{\mathrm{A}-\mathrm{B}} \simeq 1.810^{17} \mathrm{~g} \mathrm{~s}^{-1}$. The critical mass transfer rate $\dot{M}_{\mathrm{c}}$ is also slightly larger than in the model with stream impact.

The major difference with previous calculations is the presence of a critical mass transfer rate $\dot{M}_{\mathrm{SL}}$, for which one obtains a sequence of alternating short (narrow) and long (wide) outbursts with roughly the same amplitudes (see Fig. 6). At low mass transfer rates only short outbursts are present whereas for mass transfer rates higher than $\dot{M}_{\mathrm{SL}}$ all outbursts are long. In a small range of mass transfer rates outbursts of both durations are present (see Fig. 7). We return to this point in the next section.

$\dot{M}_{\mathrm{SL}}$ is comparable to $\dot{M}_{\mathrm{A}-\mathrm{B}}$; for the parameters used in Fig. $6, \dot{M}_{\mathrm{SL}}<\dot{M}_{\mathrm{A}-\mathrm{B}}$, and short outbursts are all of

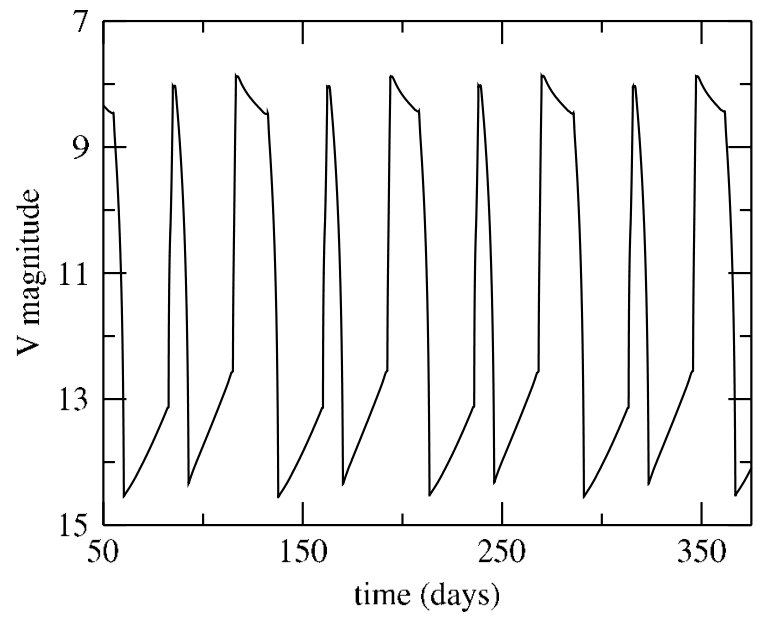

Fig. 6. Light curve for the DIM plus tidal dissipation (without stream impact) for $\dot{M}_{2}=1.510^{17} \mathrm{~g} \mathrm{~s}^{-1}$ and binary parameters of SS Cyg. Both short (narrow) and long (wide) outbursts are of inside-out type

the inside-out type. They are similar to those produced in the standard model, except that the quiescent flux is slightly larger as a result of viscous dissipation in the outer disc (as in the case of stream impact). As for the streamimpact heating case, the outburst peak luminosity is very slightly lower than in the standard model for the same mass transfer rate. The recurrence time scales are not affected by tidal dissipation when only short outbursts are produced. Long outbursts are slightly brighter than the short ones as observed (Oppenheimer et al. 1998), and for this set of parameters they can be either of the inside-out or outside-in type because the critical mass-transfer rate above which they occur $\dot{M}_{\mathrm{SL}}$ is less than $\dot{M}_{\mathrm{A}-\mathrm{B}}$.

Long outbursts appear because near the outburst maximum the outer radius increases, so does tidal dissipation. Tidal dissipation lowers the value of $\Sigma_{\min }$ so that it takes longer to reach this critical density at which a cooling-front starts to propagate and shuts off the outburst. Outbursts therefore last longer than in the standard case and have the characteristic "flat top" shape. The amplitude of the outer-disc variations increases with the mass-transfer rate. At low mass-transfer rate these variations are too small to produce an observable effect on the outburst properties. The critical rate $\dot{M}_{\mathrm{SL}}$ corresponds to the case when the disc expansion begins to affect the outburst duration. Short outburst follow long ones because the tidal dissipation lowers both the critical and the actual post-outburst surface-density so that during the next outburst the time to empty the disc is shorter. Sequences of alternating short and long outbursts are present for rather narrow interval of mass-transfer rates. At higher mass-transfer rates outbursts are of the outside-in type and therefore long (see Hameury et al. 1998).

Compared to the standard case, the recurrence time is increased when long outbursts are present, but the duration of quiescence is unchanged: the duration of the outburst is the only modified quantity. 
When stream impact heating and the tidal dissipative process are both included in the model, their effects cumulate: $\dot{M}_{\mathrm{SL}}, \dot{M}_{\mathrm{A}-\mathrm{B}}$ and $\dot{M}_{\mathrm{c}}$ are smaller by about $15 \%$ than what one gets when including only one effect (see Fig. 4 for $\dot{M}_{\mathrm{A}-\mathrm{B}}$ and $\dot{M}_{\mathrm{c}}$ ).

\section{The nature of long and short outbursts}

We have therefore a natural explanation of the coexistence of short and long outbursts in long period $(>3 \mathrm{hr}$ ) systems: for which the mass transfer is large enough for the additional heating to produce this effect; in short period system, $\dot{M}_{2}$ corresponds to short outbursts only. Heating by stream impact and tidal-torque dissipation account also for the occurrence of outside-in outbursts in SS Cyg, provided that the mass transfer rate happens to be large enough (approximatively two to three times larger than average).

In many cases, a bimodal distribution of the outbursts is observed (van Paradijs 1983). In the case of SS Cyg, Cannizzo \& Mattei (1992) identified short outbursts with a duration between 5 and 10 days, and long ones with a duration between 13 and 17 days. Cannizzo (1993) obtained in his model a sequence of alternating short and long outbursts, but this was due to the use of a fixed outer radius which is an incorrect ("brick wall") boundary condition (Hameury et al. 1998; Smak 1998). In any case his sequence looks different from the observed one and all his outbursts are inside-out (type B).

When tidal dissipation is included, one obtains, for a SS Cyg-like system, an alternating sequence of narrow and wide outbursts for a mass transfer rate $\dot{M}_{2} \sim \dot{M}_{\mathrm{SL}} \sim$ $1.510^{17} \mathrm{~g} \mathrm{~s}^{-1}$ (the value used in Fig. 6). As the short/long sequence of outbursts is not uncommon, a small mass transfer rate variation around $\dot{M}_{\mathrm{SL}}$ is the most likely cause for the occurrence of both narrow and wide outbursts. Moreover, if one believes that both A and B outburst types occur in SS Cyg (or another long period system), $\dot{M}_{2}$ must also vary around $\dot{M}_{\mathrm{A}-\mathrm{B}}$.

The value of $\dot{M}_{\mathrm{SL}}$ depends on the binary parameters and for a given orbital period one obtains a small range of mass transfer rates for which both short and long outbursts occur. Figure 7 shows this range as a function of the orbital period for a standard dwarf nova with a primary mass $M_{1}=0.6 M_{\odot}$ and a main-sequence secondary mass $M_{2}=0.11 P_{\mathrm{hr}} M_{\odot}$. Note that now the primary's mass is different from the values used previously. $M_{1}=0.6 M_{\odot}$ corresponds to the average white-dwarf mass in CVs. As mentioned earlier, $<r_{\text {out }}>$ is taken from a table in Paczyński (1977). The lower boundary of this range is $\dot{M}_{\mathrm{SL}}$ which varies by a factor of about 4 from a 2 hour period to a 7 hour period system. For short period systems, the mass transfer rate required to obtain both narrow and wide outburst is larger than $210^{16} \mathrm{~g} \mathrm{~s}^{-1}$. This is much larger than the expected secular values of a few times $10^{15} \mathrm{~g} \mathrm{~s}^{-1}$ and can explain why narrow and wide outburst alternation is not observed in short period dwarf novae. Superoutbursts in SU UMa's, which are dif- ferent from long outbursts (Warner 1995; Smak 2000), occur for mass transfer rates lower than $\dot{M}_{\mathrm{SL}}$. On the other hand there exist a subclass of the SU UMa stars, the ER UMa systems, which have very short supercycles, and are in outburst most of the time. They are presumed to have mass transfer rates as large as $10^{16} \mathrm{~g} \mathrm{~s}^{-1}$, of order of or above the value of $\dot{M}_{\mathrm{SL}}$ corresponding to their orbital periods, and should therefore show the alternation of long and short outbursts described here. This effect could add to the enhancement of the mass-transfer rate resulting from the illumination of the secondary that also cause sequences of long and short outbursts in short period systems (Hameury et al. 2000).

Figure 7 shows also $\dot{M}_{\mathrm{A}-\mathrm{B}}$; in this case we find that $\dot{M}_{\mathrm{SL}}$ is larger than $\dot{M}_{\mathrm{A}-\mathrm{B}}$, which means that all long outbursts are now of the outside-in type. However, the resulting light curves in which an alternance of short and long outbursts is found do not differ much from the one shown in Fig. 6, except that the rise times are shorter.

We propose that stochastic variations of the masstransfer rate for the secondary cause the sequence of alternating short and long, as well as type A and type B outbursts. For the parameters of SS Cyg, we need masstransfer rate variations in the range $\sim 1-210^{17} \mathrm{~g} \mathrm{~s}^{-1}$ to obtain the four types in the same light curve; this range depends on the system parameters, and in particular on the orbital period (see Fig. 7), and on the mass of the primary. Because outburst duration depends mainly on the disc's size (Smak 1999b) for short outbursts whose duration is independent of the mass transfer rate we obviously reproduce the correlation (van Paradijs 1983) between the outburst width and the orbital period. It is much more difficult to reproduce the observed correlation for long outbursts, since their duration is quite sensitive to $\dot{M}_{2}$, whose variation with the orbital period is not well known. One must, however, be cautious when interpreting this correlation, as (i) the statistics is small, and (ii) this correlation may result in part from the very definition of "long" outbursts: they must last longer than short outbursts, whose duration increases with the orbital period simply because of the increase of the disc's size (Smak 1999b). In the model by Smak (1999a), long outbursts have to be interrupted by $\dot{M}_{2}$ returning to a low value since otherwise the disc would remain steady. This requires the action of an external physical mechanism with an unspecified free parameter. If for long outbursts the correlation is real, in Smak's model the mechanism terminating long outbursts would have to depend on the orbital period.

Since $\dot{M}_{\mathrm{SL}}$ is much larger than the mass-transfer rates expected in systems below the period gap, we do not expect long outbursts for periods shorter than 2 hours. This agrees with observations. Superoutbursts observed in SU UMa systems must have a different explanation; they are either due to a tidal instability, as proposed by Osaki (1996), and/or to the irradiation of the secondary, which is quite efficient in these short period systems (Hameury et al. 2000). 


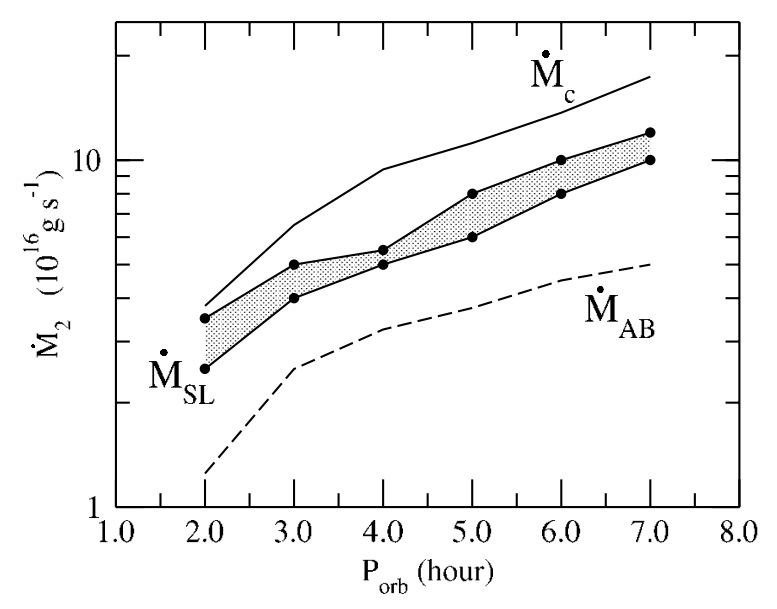

Fig. 7. Range of mass transfer rates for which both long and short outbursts occur and critical rate, as a function of the orbital period. Here, $M_{1}=0.6 M_{\odot}$ and $M_{2}=0.11 P_{\text {orb }} M_{\odot}$. The critical rate $\dot{M}_{\mathrm{c}}=9.510^{15} \dot{M}_{1}^{-0.89} r_{10}^{2.68}$ (see Hameury et al. 1998). For low $\dot{M}$, only short outbursts are observed; above the upper curve, all outbursts are long. The upper solid line shows the critical mass transfer rate above which the disc is stable

We still cannot, however, account for the presence of outside-in outbursts in short period systems, whereas normal outbursts in SU UMa systems are generally believed to be of this type (Osaki 1996). There are two possible ways out of this difficulty: first, as discussed in the Sect. 6, SU UMa normal outbursts occur in truncated discs and could be of the inside-out type; second, the disc outer radius could be much smaller than what the standard DIM predict (Smak 2000); indeed, observations of eclipsing dwarf novae by Harrop-Allin \& Warner (1996) tend to substantiate this idea. The second option is also favoured by the "direct" observations of outside-in outbursts in two eclipsing SU UMa systems (see Sect. 6.2 for references).

\section{Inside-out versus outside-in outbursts}

Outbursts of SS Cyg are believed to be of both A and B types (Smak 1984; Cannizzo et al. 1986; Mauche 1996; Smak 1998). Thus, the mass transfer rate from the secondary probably varies around the A-B transition. In models, outside-in outbursts are usually obtained only for mass transfer rates large compared to those expected from binary evolution models (Baraffe \& Kolb 1999). Moreover, disc model computations with such values of $\dot{M}_{2}$ predict recurrence times that are much shorter than observed. Ichikawa \& Osaki (1992) managed to get rid of this problem by using an $r$-dependent $\alpha$-prescription specially designed to suppress type B outbursts. Their viscous diffusion time is then almost independent of the radius and inside-out outbursts occur only at very small mass transfer rates.

As mentioned earlier, including the stream-impact heating lowers $\dot{M}_{\mathrm{A}-\mathrm{B}}$ without changing the $\alpha$-prescription. We obtain $\dot{M}_{\mathrm{A}-\mathrm{B}}=1.710^{17} \mathrm{~g} \mathrm{~s}^{-1}$, 1.4 times smaller than in the standard case; this is reasonably close to estimates of SS Cyg average mass transfer rate $\dot{M}_{2}=$ $610^{16} \mathrm{~g} \mathrm{~s}^{-1}$ (e.g. Patterson 1984). An increase of the mass transfer rate by a factor $2-3$ is therefore sufficient to provoke outside-in outbursts. On the other hand, for short period systems, the transition between inside-out and outside-in outburst still occurs for much too large mass transfer rates $\left(210^{16} \mathrm{~g} \mathrm{~s}^{-1}\right)$, whereas estimates of mass transfer in these systems do not exceed a few $10^{15} \mathrm{~g} \mathrm{~s}^{-1}$ (Warner 1995; Baraffe \& Kolb 1999).

\subsection{Predictions of the DIM}

Outside-in outbursts occur if the time scale for matter accumulation at the outer disc edge $t_{\text {accum }}$ is shorter than the viscous drift time scale $t_{\mathrm{drift}}$. These times are (Osaki 1996):

$$
\begin{aligned}
& t_{\text {accum }}=\frac{4 \pi^{2} r^{2} \nu \Sigma_{\max }^{2}(r)}{\dot{M}^{2}} \\
& t_{\text {drift }}=r^{2} \delta \nu^{-1}
\end{aligned}
$$

where $\nu=2 / 3 \alpha(\mathcal{R} / \mu) T / \Omega$ is the kinematic viscosity, and $\delta \sim 0.05$ is a numerical correction factor. Using the analytical fits of $\Sigma_{\max }$ given by Hameury et al. (1998), the condition for outside-in outbursts is:

$$
\dot{M}>4.3510^{15} \alpha_{\mathrm{c}}^{0.17}\left(\frac{T}{4000 \mathrm{~K}}\right) M_{1}^{-0.88} r_{10}^{2.64} \mathrm{~g} \mathrm{~s}^{-1}
$$

where $r_{10}$ is the radius measured in $10^{10} \mathrm{~cm}$ and where we have assumed $\mathcal{R} / \mu=510^{7}$. This condition can be compared with our numerical results. In the case without the additional effects, our critical rate is $2.510^{17} \mathrm{~g} \mathrm{~s}^{-1}$ for long period systems, whereas Eq. (9) gives $1.810^{17} \mathrm{~g} \mathrm{~s}^{-1}$; for short period systems, these two numbers are $2.510^{16}$ and $2.810^{16} \mathrm{~g} \mathrm{~s}^{-1}$ respectively. It must be noted that the quality of the agreement should not be a surprise, since the quantity $\delta$ was introduced by Osaki (1996) precisely for obtaining good fits.

When heating of the outer parts of the disc is included Eq. (9) is no longer valid. Osaki (1996) assumed that in quiescence the surface density is parallel to the critical density profiles, that $\Sigma \sim 2 \Sigma_{\min }$, and he used the standard critical densities. Things are different when heating of the outer disc is included. Near the disc outer rim both $\Sigma_{\min }$ and $\Sigma_{\max }$ are considerably reduced as is the distance between them. These effects reduce the value of $\dot{M}_{\mathrm{A}-\mathrm{B}}$.

This reduction is not sufficient to obtain desired values of the mass-transfer rate, i.e. to $\sim 310^{15} \mathrm{~g} \mathrm{~s}^{-1}$, which is typical of the mass transfer rate in SU UMa systems. The only hope is in the strong dependence of $\dot{M}_{\mathrm{A}-\mathrm{B}}$ on the outer disc radius. The mean radius we have assumed for short period systems $\left(2.310^{10} \mathrm{~cm}\right)$ is probably too large for most SU UMa's since it exceeds in many cases the 3:1 resonance radius, which is of order of $0.46 a$, i.e. $210^{10} \mathrm{~cm}$ for a total mass of $0.7 M_{\odot}$ and a period of $1.6 \mathrm{hr}$ characteristic of SU UMa stars. The 3:1 resonance radius should be reached only during superoutbursts and should be much 
smaller $\left(0.3 a\right.$, i.e. $\left.1.310^{10} \mathrm{~cm}\right)$ during the first few outbursts of a supercycle. For such small radii $\dot{M}_{\mathrm{A}-\mathrm{B}}$ would then be reduced by a factor $\sim 4-5$, i.e. to a value slightly larger than the estimated mass transfer rate in SU UMa systems.

In Fig. 8 we show the quiescence time and the radius at which the outburst starts versus mass transfer rate, for a short period system with a mean outer disc radius $\sim 1.310^{10} \mathrm{~cm}$. This model corresponds to a relaxed disc, i.e. a disc whose mass is constant over an outburst cycle. Such small discs are expected to exist only during the first half of a supercycle and later should grow in size. We have assumed a large coefficient $c$ in Eq. (4), in order to obtain a small disc, but we have not included heating by tidal torques that would have resulted in much too large a dissipation: the dissipation of tidal torques must be negligible in discs much smaller than the truncation radius. As can be seen, outside-in outbursts occur for mass transfer rates as low as $5-610^{15} \mathrm{~g} \mathrm{~s}^{-1}$, reasonably close to the expected value in these systems.

It is worth noting that the recurrence time depends on the mass transfer rate, even in the case of inside-out outbursts. A comparison of Figs. 4, 5 and 8 shows that this effect is related to the size of the disc. This is due to the fact that a local dimensional analysis becomes invalid when the radius of the outer edge of the disc is not much larger than the inner radius (for example, in the case of steady discs, the inner boundary condition introduces a factor $\left.\left[1-\left(r_{\text {in }} / r\right)^{1 / 2}\right]\right)$. Osaki (1996) asserts that the correlation $t_{\mathrm{s}} \propto t_{\mathrm{n}}^{0.5}$ found between the supercycle recurrence time $t_{\mathrm{s}}$ and the recurrence time of normal outbursts $t_{\mathrm{n}}$ in SU UMa systems results from Eq. (7) and the relation $t_{\mathrm{s}} \propto \dot{M}^{-1}$ which results from the assumption that superoutburst occur when sufficient mass is accumulated (Osaki 1996; Hameury et al. 2000). Clearly, this cannot be the case if one uses the standard disc model in which $\alpha$ is not forced to adopt a functional dependence that would give the desired effect but is just kept constant. In any case, the mass transfer rate cannot be assumed to be the only variable on which recurrence times depend. For example Menou (2000) showed that both the normal outburst and super-outburst recurrence times strongly depend on the secondary to primary mass ratio.

Small discs are also expected if one reduces the index $n$ in Eq. (4), since the smaller $n$, the larger the amplitude of disc radius variations during outbursts; for a given maximum disc radius (some fraction of the Roche radius), the disc radius during quiescence is therefore expected to be smaller for smaller $n$.

\subsection{Observations}

Campbell (1934) identified four distinct types of outburst in SS Cyg light curves which have a large variety of characteristic rise times. Since then, several techniques have been used to investigate the physical outburst properties: multi-wavelength photometry, spectrometry, eclipse mapping and model fitting. The first disc instability models

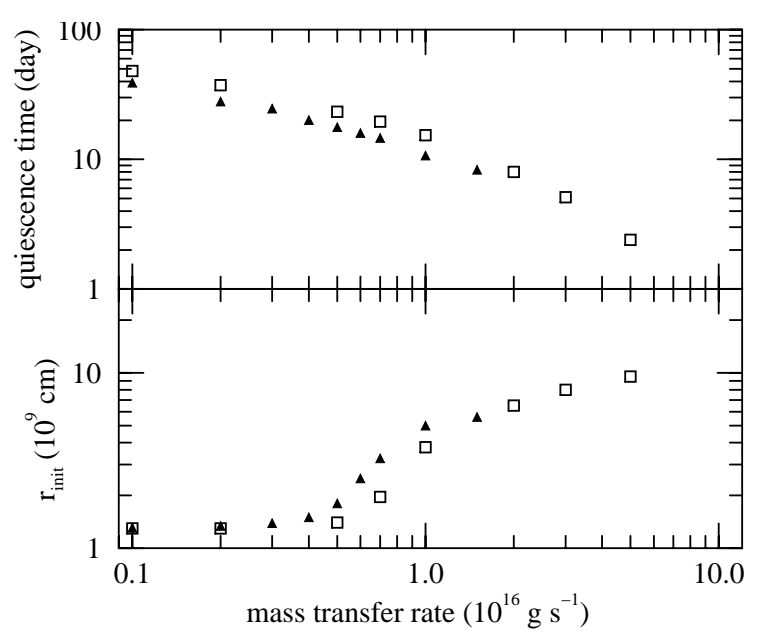

Fig. 8. Radius $R_{\text {init }}$ at which the instability is triggered and quiescent time as a function of the mass transfer rate from the secondary. The parameters are those of short period systems with $\left\langle r_{\text {out }}\right\rangle \sim 1.310^{10} \mathrm{~cm}$. Squares correspond to the standard DIM, and circles to the case where the stream impact has been included

have predicted the outburst types A (outside-in) and B (inside-out) (Smak 1984), and showed that the first category rises earlier at optical wavelengths and have a more asymmetric shape. As a result, asymmetric outbursts are usually supposed to be of type A. However asymmetric outbursts can also be of the inside-out type. For instance, in Fig. 6, long outbursts are not symmetric but are of type B.

The UV delay is the observed lag between the rise in optical and the rise in UV. Its duration is believed to be a signature of the outburst starting region. In the naive picture since the hottest parts of the disc associated with the shortest wavelengths are close to the white dwarf, there should be almost no UV delay if the outburst starts in the inner part of the accretion disc, whereas one should expect an important UV delay for outside-in outbursts. However, as discussed by Smak (1998), things are more complicated than what the naive picture would suggest, and long delays can also exist for inside-out outbursts if the outer disc radius expansion during outburst and the correct size of the disc are taken into account. For a given binary system the UV delay will always be longer for an outside-in outburst than for an inside-out one, but the length of the delay is not sufficient by itself to determine the outburst type.

Smak (1998) proposed that the ratio of the UV delay and the outburst duration is a good indicator of the outburst type. However, the existence of both long and short outbursts at a given orbital period makes this test very uncertain. Indeed Fig. 6 shows a probing counter-example where both short and long outbursts are of type B and for which Smak's ratio would be quite different.

In addition Hameury et al. (1999), using SS Cyg parameters, reproduced the observed (Mauche 1996) asymmetric outburst and 1-day EUV delays for an inside-out 
outburst starting to propagate at the inner edge of a truncated disc. This obviously raises the question of the real relationship between the outburst type and the UV delay.

It has been known for some time that eclipse profiles allow to determine the propagation direction of an outburst (Smak 1971 for U Gem; Vogt 1984 and Rutten et al. 1992 for OY Car). For example in the case of an accretion disc whose luminosity is much larger than both the white dwarf and the hot spot luminosity, the eclipse profile is almost symmetric. This is the case of HT Cas, a SU UMa system with a period of $106 \mathrm{~min}$ (Ioannou et al. 1999). The authors found that during the rise of the burst, the eclipse is shallow and its width is large. This means that most of the flux originates from the outer parts of the disc. They observed that the width of the eclipse decreases after the outburst peak, which is in perfect agreement with the predicted decrease of the disc radius during decline, therefore proving the outside-in nature of the outburst. Webb et al. (1999) and Baptista et al. (2000) used the same technique to bring evidences of the inside-out nature of an outburst of IP Peg and EX Dra respectively.

At present, the only way to be sure of the outburst type is to use, whenever possible, eclipse profiles. As eclipses are not present in all systems it would be worthwhile to test Smak's assumption on the UV delay in eclipsing dwarf novae.

\section{Conclusions}

We have shown that the inclusion of additional heating effects such as the stream impact and tidal dissipation might solve several problems of the standard DIM.

The most important result is that when the tidal torque dissipation in the outer disc regions is included, one obtains for a certain range of mass-transfer rates an alternation of short and long outbursts which is observed in long orbital period system such as SS Cyg (Cannizzo \& Mattei 1992) or Z Cam (Oppenheimer et al. 1998). The long outbursts are slightly brighter than short outbursts as observed. Moderate fluctuations of the mass-transfer rate would extend the range for which such alternating outbursts are present. For systems with mass transfer rates close to the stability limit, such fluctuations will produce standstills as shown in Buat-Ménard et al. (2000). This solution of the long/short outburst problem (Smak 2000) is different from the one suggested by Smak (1999a). In our model mass-transfer fluctuations play only an auxiliary role and long outbursts do not result from fluctuations bringing the disc to a steady state.

We showed that additional heating effects make the outside-in outburst possible at low mass transfer rates without changing the $\alpha$-prescription. Our results on mass transfer rates and outburst types are in good agreement with most estimates in the case of long period systems. In the case of short period systems of the SU UMa type, for outside-in outburst to occur the disc would have to be smaller than usually assumed. For small discs the recurrence times of both the inside-out and outside-in outbursts is $\dot{M}_{2}$-dependent. It must be stressed out that there are still many uncertainties on the observational determination of outburst types. For instance, the UV delay is not necessarily a good criterion for determining the outburst type; the eclipse profile during outburst is a much better criterion, but unfortunately restricted to a small subset of dwarf novae.

There are still several points that need to be clarified. First, the stream impact effect geometry is not well known and is considered here in a 1D approximation (as for the tidal dissipation). We do not know how far and how efficiently the energy propagates in the disc. The expression of the tidal torque can also be subject to discussion. Moreover, we did not include irradiation here, and it would be interesting to study the combined effects of all phenomena. Finally, as raised above, the existing discrimination criteria on the outburst types $\mathrm{A}$ and $\mathrm{B}$ have to be tested and improved.

Acknowledgements. We thank Guillaume Dubus for helpful comments. This work was supported in part by a grant from Programme National de Physique Stellaire of the CNRS.

\section{References}

Armitage, P. J., \& Livio, M. 1996, ApJ, 470, 1024

Armitage, P. J., \& Livio, M. 1998, ApJ, 493, 898

Baptista, R., Catalán, M. S., \& Costa, L. 2000, MNRAS, 316, 529

Baraffe, I., \& Kolb, U. 1999, MNRAS, 309, 1034

Buat-Ménard, V., Hameury, J.-M., \& Lasota, J.-P. 2000, A\&A, submitted

Campbell, L. 1934, AHCO, 90, 93

Cannizzo, J. K. 1993, ApJ, 419, 318

Cannizzo, J. K., \& Mattei, J. A. 1992, ApJ, 401, 642

Cannizzo, J. K., Wheeler, J. C., \& Polidan, R. S. 1986, ApJ, 301,634

Dubus, G., Lasota, J.-P., Hameury, J.-M., \& Charles, P. 1999, MNRAS, 303, 139

Hameury, J.-M., Lasota, J.-P., \& Huré, J.-M. 1997, MNRAS, 287,937

Hameury, J.-M., Menou, K., Dubus, G., Lasota, J.-P., \& Huré, J.-M. 1998, MNRAS, 298, 1048

Hameury, J.-M., Lasota, J.-P., \& Dubus, G. 1999, MNRAS, 303,39

Hameury, J.-M., Lasota, J.-P., \& Warner, B. 2000, A\&A, 353,244

Harrop-Allin, M. K., \& Warner, B. 1996, MNRAS, 279, 228

Ichikawa, S., \& Osaki, Y. 1992, PASJ, 44, 15

Ichikawa, S., \& Osaki, Y. 1994, PASJ, 46, 621

Ioannou, Z., Naylor, T., Welsh, W. F., et al. 1999, MNRAS, 310,398

Lasota, J.-P. 2000, New Astronomy Reviews, submitted

Lasota, J.-P., Hameury, J.-M., \& Huré, J.-M. 1995, A\&A, 302, L29

Livio, M., \& Pringle, J. 1992, MNRAS, 259, 23

Lubow, S. H., \& Shu, F. H. 1975, ApJ, 198, 383

Marsh, T. R., \& Horne, K. 1990, ApJ, 349, 593

Mauche, C. W. 1996, in Astrophysics in the Extreme Ultraviolet, ed. S. Bowyer, \& R. F. Malina (Dordrecht, Kluwer), 317. 
Menou, K. 2000, Science, in press

Meyer, F., \& Meyer-Hofmeister, E. 1983, A\&A, 121, 29

Meyer, F., \& Meyer-Hofmeister, E. 1994, A\&A, 228, 175

Oppenheimer, B. D., Kenyon, S. J., \& Mattei, J. A. 1998, AJ, 115,1175

Osaki, Y. 1985, A\&A, 144, 369

Osaki, Y. 1989, PASJ, 41, 1005

Osaki, Y. 1996, PASP, 108, 39

Paczyński, B. 1977, ApJ, 216, 822

Papaloizou, J., \& Pringle, J. E. 1977, MNRAS, 181, 441

Patterson, J. 1984, ApJS, 54, 443

Ritter, H., \& Kolb, U. 1998, A\&AS, 129, 83

Rutten, R. G. M., van Paradijs, J., \& Tinbergen, J. 1992, A\&A, 260,213

Shakura, N. I., \& Sunyaev, R. A. 1973, A\&A, 24, 337
Smak, J. 1971, Acta Astr., 21, 15

Smak, J. 1984, Acta Astron., 34, 161

Smak, J. 1998, Acta Astron., 48, 677

Smak, J. 1999a, Acta Astron., 49, 383

Smak, J. 1999b, Acta Astron., 49, 391

Smak, J. 2000, New Astronomy Review, 44, 171

Spruit, H. C., \& Rutten, R. G. M. 1998, MNRAS, 299768

Stehle, R., \& King, A. R. 1999, MNRAS, 304, 698

van Paradijs, J. 1983, A\&A, 125, L16

Vogt, N. 1983, A\&A, 128, 29

Warner, B. 1994, Ap\&SS, 226, 187

Warner, B. 1995, Cataclysmic Variable Stars (Cambridge University Press, Cambridge)

Webb, N. A., Naylor, T., Ioannou, Z., et al. 1999, MNRAS, 310,407 